\title{
Clasificación en subtipos moleculares de tumores de mama de pequeños animales mediante métodos inmumohistoquímicos
}

\author{
Ortega García Mª. ${ }^{1}$, Galán Torres JA. ${ }^{2}$, Millán Ruiz Y. ${ }^{3}$, Sánchez Céspedes R. ${ }^{3}$, \\ Martín de las Mulas González-Albo J. ${ }^{3}$
}

Sanid. mil. 2013; 69 (1): 6-12; ISSN: 1887-8571

\begin{abstract}
RESUMEN
Antecedentes y Objetivos: Aplicar un panel de anticuerpos (anti -receptor de progesterona, -receptor de estrógenos, -receptor del factor de crecimiento epidérmico humano 2 y -citoqueratina 14) utilizando métodos inmunohistoquímicos en tumores mamarios de pequeños animales para analizar su clasificación en subtipos moleculares y su asociación con la invasión, el grado y el tipo histológico de las neoplasias. Material y Métodos: Muestras de tumores mamarios malignos, 10 de la especie canina y 3 de la felina. Control positivo interno: glándula mamaria no tumoral adyacente a las neoplasias. Resultados: El 23\% (3/13) de los tumores fueron del subtipo luminal B, el 23\% (3/13) fueron HER2 positivos, el 46\% (6/13) fueron basales y el 7,6\% (1/13) no se pudieron clasificar porque no expresaron ninguno de los marcadores tumorales analizados. Ningún caso fue del subtipo luminal A. Los 6 tumores basales fueron de grado II o III y presentaban o infiltración de solo el estroma o también invasión vascular. Dos tercios de los tumores HER2 positivos presentaban infiltración del estroma y 1/2 tumores resultó ser de grado II. Los tumores luminal B, 2/3 fueron de grado II o III. Todos los controles internos fueron positivos. No se encontraron diferencias significativas en la distribución de los subtipos moleculares entre los diferentes grupos de las variables invasión ( $\mathrm{p}$-valor=0,26), ni grado de malignidad ( $\mathrm{p}$-valor=0,42). Sí hubo diferencias en el límite de la significación estadística en la distribución de los subtipos moleculares entre los diferentes grupos de la variable tipo histológico ( $p=0,08)$. Conclusiones: La aplicación del panel de anticuerpos ha permitido descubrir 4 (luminal B, HER2, basal y sin clasificar) de los 5 subtipos moleculares posibles.
\end{abstract}

PALABRAS CLAVE: Receptor. Subtipo molecular. Método inmunohistoquímico.

\section{Classification in molecular subtypes of breast tumors of small animals through immunohistochemical methods}

SUMMARY: Antecedents and objectives: to apply an antibodies panel (anti-progesterone receptor -estrogen receptor, -human epidermal growth factor receptor 2 and cytokeratin 14) using immunohistochemical methods in mammary tumors of small animals to classify them in molecular subtypes and their association with the invasion, grade and histological type of the malignancies. Material and Methods: samples of malignant mammary tumors, 10 from canine species and 3 from feline ones. Internal positive control: nontumoral mammary gland adjacent to the malignancy. Results: 23\% (3/13) of the tumors were of the luminal B subtype, 23\% (3/13) were HER2 positive, 46\% (6/13) were basal types and 7,6\% (1/13) were unclassifiable because they did not express any of the tested tumor markers. None of the cases belonged to the luminal A subtype. The 6 basal tumors were grade II or III and presented only stromal infiltration or vascular invasion as well. Two thirds of the HER2 positive tumors presented stromal infiltration and half the tumors were grade II. Two thirds of luminal B tumors were grade II or III. All internal controls were positive. There were no significant differences in the distribution of the molecular subtypes among the different groups of the invasion $(p$-value $=0.26)$ and malignancy grade variables ( $p$-value $=0.42$ ). There were differences of borderline statistical significance in the distribution of the molecular subtypes among the different groups of the histological type variable ( $p$-value $=0.08$ ). Conclusions: the application of the antibodies panel has allowed to find 4 (luminal B, HER2, basal and unclassified) out of 5 possible molecular subtypes.

KEY WORDS: Receptor. Molecular subtype. Immunohistochemical method.

\section{INTRODUCCIÓN}

En las glándulas mamarias normales de las especies humana, canina y felina, los conductos y alveolos están rodeados por dos capas de células, una interna o luminal de células secretoras y

\footnotetext{
Cap. Veterinario. Escuela Militar de Sanidad. Madrid. España.

2 Tcol. Veterinario. Centro Militar de Veterinaria de la Defensa. Servicio de Microbiología, Higiene y Sanidad Ambiental. Madrid. España.

Universidad de Córdoba. Facultad de Veterinaria. Departamento de Anatomía y Anatomía Comparadas. Córdoba. España.
}

Dirección para correspondencia: Juan Alberto Galán Torres. Centro Militar de Veterinaria de la Defensa. C/ Darío Gazapo N. ${ }^{\circ} 3$. CP 28024. Madrid. España. Teléfono: 915 122496 - 915122 475. Fax: 915122 490. Correo electrónico: jgaltor@oc.mde.es

Recibido: 12 de julio de 2012

Aceptado: 31 de octubre de 2012 otra externa claramente diferente, pegada a la membrana basal, denominada capa basal/mioepitelial. En estas especies, las células epiteliales luminales se caracterizan por la expresión de citoqueratinas ( $\mathrm{CK}$, en adelante) luminales de bajo peso molecular, que incluyen CK8, CK18, CK19 y CK $7^{1-4}$. La capa externa de células está formada por células que expresan de forma variable citoqueratinas basales de alto peso molecular como CK5, CK6, C14 y CK17 junto a otros marcadores como actina de músculo liso, calponina, vimentina y $\mathrm{p} 63^{5-9}$

Durante la transformación celular y el desarrollo tumoral, la mayoría de los marcadores de diferenciación específicos parece que se conservan ${ }^{10,11}$. Esta propiedad hace posible que estas proteínas se usen como marcadores tumorales para los tumores mamarios de la especie humana y de los carnívoros domésticos. 


\section{Clasificación en subtipos moleculares de tumores de mama de pequeños animales mediante métodos...}

Las características epidemiológicas, clínicas y morfológicas de los tumores de mama son diferentes en las hembras de las especies domésticas canina y felina.

La edad de presentación de los tumores mamarios en la especie canina está comprendida entre los 10 y los 11 años. La tasa de incidencia por 100.000 individuos es de 111 por año ${ }^{12}$. La mitad de los tumores mamarios en la perra son malignos y los tipos que predominan son carcinoma simple, carcinoma anaplásico, sarcoma y carcinosarcoma ${ }^{13}$. No existe predisposición racial, aunque en algunos trabajos se citan razas como Caniche, Spaniel bretón, Setter inglés, Pointer, Fox terrier, Boston terrier, Cocker spaniel y Lhasa apso.

Los tumores de mama en la especie felina se presentan entre los 10 y los 14 años de edad. Su incidencia por 100.000 hembras no castradas es de 31,8 por año ${ }^{14}$. Entre el 80 y el $96 \%$ de los tumores mamarios son malignos y el tipo más frecuente es el adenocarcinoma ${ }^{15}$. La raza siamesa parece tener un mayor riesgo.

El cáncer de mama en la especie humana se considera una enfermedad heterogénea, y se clasifica mediante un perfil de expresión génica en dos tipos según la presencia o ausencia de receptores hormonales (estrógenos y/o progesterona). Dentro de cada tipo, a su vez, se han establecido diferentes subtipos, dos para los positivos a receptores hormonales (luminal A y luminal B) y tres para los negativos a receptores hormonales (el que expresa el receptor 2 del factor de crecimiento epidérmico humano o HER2 $2^{(1)}$, el basal y el no clasificado) $)^{16,17}$.

Estudios posteriores han demostrado que estos subtipos se conservan a lo largo de diferentes series de pacientes y de plataformas de chips genéticos ${ }^{(2)}$, y que los factores de predicción del resultado clínico de las pacientes basados en la expresión de diferentes genes siguen probablemente un mismo grupo de subtipos biológicos, ya que coinciden de forma significativa ${ }^{18,19}$.

Por otro lado, se han propuesto también paneles de anticuerpos con técnicas inmunohistoquímicas que podrían usarse en lugar del perfil de expresión génica para identificar los grupos moleculares.

El coste y la complejidad de los perfiles de expresión genética hacen impracticable su uso en laboratorios equipados para tests diagnósticos de rutina. Sin embargo, alguno de los paneles inmunohistoquímicos propuestos podrían identificar los grupos basados en tipos moleculares y clasificarlos siguiendo un diagrama de flujo ${ }^{20}$.

Los paneles contienen al menos anticuerpos anti-receptor de estrógenos (RE, en adelante), anti-receptor de progesterona (RP, en adelante), anti-HER2 y anti-citoqueratinas basales (CK5/6 y $\mathrm{CK} / 14)^{21,22}$.

Los RE y RP están presentes de forma natural en el tejido mamario, persisten en lesiones benignas de tipo displásico, hiperplásico o neoplásico benigno, pero disminuyen en densidad o desaparecen en neoplasias malignas. El RE alfa se expresa en los tejidos de la mama (células epiteliales de alveolos y conductos), útero y vagina. El RP se expresa también en células epiteliales alveolares y ductales de la mama, en endometrio, epitelio cervical y en células lisas miometriales.

(1) El receptor 2 del factor de crecimiento epidérmico aparece en la literatura científica bajo diferentes denominaciones, como HER2, c-ERB-2, neu, ERB-B2. Este estudio usa el acrónimo HER2.

(2) Un ADN array (también denominado ADN micro-array, ADN chip, oligonucleotide array o gene chip). Busquets X y Agustí AGN. Chip genético (ADN array): el futuro ya está aquí. Arch Bronconeumol 2001; 37: 394-396.

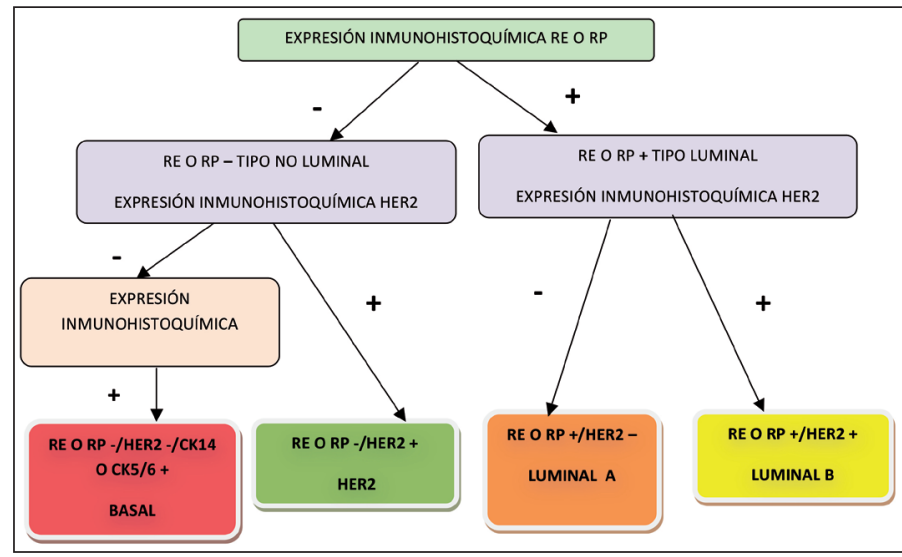

Figura 1. Diagrama de flujo de clasificación en subtipos moleculares utilizando anticuerpos y técnicas inmunoquímicas.

El HER2 es un protooncogen que codifica un receptor tirosin-quinasa, concretamente una glicoproteína transmembranosa similar al receptor del factor de crecimiento epidérmico conocido como proteína HER2, que ha sido descrito en diferentes tumores y animales. El HER2 se expresa en adenocarcinomas de diferentes localizaciones, incluyendo ovario, tracto gastrointestinal y mama. También se expresa en carcinomas de células transicionales de vías urinarias.

Los tumores de mama basales se caracterizan por la pérdida de la expresión de RE, RP y HER2 y por la expresión de citoqueratinas $5 / 6$ y 14 , mientras que el subtipo luminal se caracteriza por la expresión de RE o RP, y se puede dividir en luminal A o luminal B dependiendo de la ausencia o presencia de expresión de HER2. La negatividad para los marcadores luminales, la positividad o negatividad para los marcadores basales y la positividad a HER2 caracteriza al grupo que sobreexpresa HER2 20-22.

Los tumores de mama en pequeños animales, se consideran un modelo espontáneo del cáncer de mama de la especie huma$\mathrm{na}^{23,24}$ basándose frecuentemente en las características histomorfológicas, pero respecto a la perra, existen algunas diferencias entre los tumores de las dos especies que deberían tenerse en cuenta a la hora de hacer comparaciones. La proliferación de células mioepiteliales es un hallazgo frecuente en los patrones denominados mixtos y complejos, pero no es una característica frecuente del cáncer de mama humano ${ }^{25,26}$. Además, es frecuente la presencia de tumores múltiples en la primera presentación clínica en el caso de tumores de mama en la especie canina, pero no en la humana ${ }^{26,27}$. Por lo tanto, la clasificación en grupos moleculares, recientemente adoptada para el cáncer de mama en la especie humana, parece ser una mejor herramienta que las características morfológicas para establecer similitudes entre los dos modelos.

En Veterinaria, tres recientes investigaciones ${ }^{28-30}$ han tratado de aplicar a los carcinomas mamarios caninos paneles de anticuerpos similares con técnicas inmunohistoquímicas pero la existencia de todos los subtipos inmunohistoquímicos encontrados en los tumores de mama en la especie humana y la diversidad en su comportamiento biológico no ha sido probada hasta la fecha. Los perros con tumores luminales, que son normalmente de bajo grado y bajo índice de proliferación, tienen un buen pronóstico con un intervalo largo libre de enfermedad y supervivencia. Cuando este perfil inmunohistoquímico se pierde, 
los tumores muestran características histológicas agresivas y se acortan el intervalo libre de enfermedad y la supervivencia posquirúrgica. Sin embargo, existen datos contradictorios respecto a los tumores HER2 positivos y sobre la definición y comportamiento biológico de los basales. Los investigadores usan diferentes paneles para marcadores basales y establecen diferentes criterios para definir qué constituye positividad, lo que puede explicar las discrepancias entre los resultados ${ }^{28,30}$.

La expresión inmunohistoquímica de RE en los tumores mamarios caninos varía según los autores entre el 50,6\% $\%^{31}$ y el $66 \% 32$. El tamoxifeno, bloquea la acción de los estrógenos que estimulan el desarrollo de las células tumorales, y se utiliza como terapia complementaria en el tratamiento de los cánceres que expresan receptores de estrógenos en la especie humana.

La expresión inmunohistoquímica de RP en los tumores mamarios caninos varía también según los mismos autores entre el $66 \% \%^{32}$ y el $71,9 \% \%^{31}$. Los tumores malignos que expresan RP presentan supervivencias significativamente más largas, sobre todo cuando existe positividad combinada para RE y RP ${ }^{31}$.

En la especie humana, la sobreexpresión de HER2 se relaciona con caracteristicas clinicopatológicas más agresivas y sensibilidad a quimioterapia específica (trastuzumab, Herceptin ${ }^{\circledR}$ ) en cáncer de mama ${ }^{33,34}$. Para la sobreexpresión del HER2 en los tumores mamarios caninos, los resultados de diferentes estudios son contradictorios, pero varían del 18-35\% $\%^{35,36}$. Otro estudio encontró un porcentaje del 17,6\% $\%^{37}$. Para los tumores de mama en la gata la sobreexpresión de HER2 varía del $36 \%$ al 90\% $0^{38-40}$. En otro estudio diferente se obtuvo un porcentaje de sobreexpresión en carcinomas de gata del $40 \% 0^{41}$. La alteración del protooncogen se ha descrito en los tumores de mama de gatas $^{38-40}$ y perras ${ }^{35-37,42}$ la mayoría a nivel de proteína. Los carcinomas de mama en la gata con sobreexpresión de HER 2 tienen características indicativas de gran malignidad que incluyen gran tamaño, grado histológico alto y ausencia de receptores hormonales ${ }^{41,43}$.

Los tumores mamarios caninos y felinos constituyen un modelo animal espontáneo del cáncer de mama en la especie humana.

La clasificación del cáncer de mama de la especie humana basada en subtipos moleculares es una herramienta más útil que las características morfológicas de los tumores para establecer similitudes entre los dos modelos.

\section{Objetivos}

Con este trabajo se pretende aplicar un panel de anticuerpos (anti -RP, -RE, -HER2 y -CK14) utilizando métodos inmunohistoquímicos en tumores mamarios de pequeños animales para analizar su clasificación en subtipos moleculares y su asociación con la invasión, el grado y el tipo histológico de las neoplasias.

\section{MATERIAL Y MÉTODOS}

\section{Sujetos de estudio, periodo de estudio y origen de las muestras}

Para la realización de este estudio se han utilizado 13 muestras de tumores mamarios malignos, 10 de la especie canina y 3 de la felina.
Las razas caninas de los casos han sido Cocker spaniel, Pastor alemán, Caniche y Setter irlandés, mientras que sólo ha habido una raza felina, Común europeo.

Las edades de las perras han estado comprendidas entre 7-11 años y la de las gatas entre 7-9 años.

Los casos clínicos corresponden a los remitidos al Servicio de Microbiología, Higiene y Sanidad Ambiental durante el primer semestre de 2012 y los procedentes del archivo del servicio entre 2006-2011.

El diagnóstico histológico y la valoración de la invasión, el grado y tipo histológico se han realizado en el Centro Militar de Veterinaria de la Defensa (CEMILVETDEF, Madrid), mientras que las técnicas inmunohistoquímicas, la valoración de sus resultados y un segundo diagnóstico histológico por otro patólogo experimentado se realizaron en el Departamento de Anatomía y Anatomía Patológica Comparadas-Facultad de Veterinaria de Córdoba.

Se han realizado 5 secciones seriadas por muestra.

Como control positivo para las técnicas inmuohistoquímicas se ha utilizado la expresión de los marcadores analizados en la glándula mamaria no tumoral adyacente a las neoplasias (control positivo interno).

\section{Métodos}

\section{Procesamiento de las muestras}

En primer lugar, las muestras han sido procesadas para su tinción con hematoxilina y eosina. En segundo lugar, se han realizado las técnicas inmunohistoquímicas que se describen a continuación para cada uno de los anticuerpos usados en el estudio. Para ello se han seguido el seminario de técnicas inmunohistoquímicas impartido en el Centro Militar de Veterinaria de la Defensa ${ }^{44}$ y el protocolo utilizado por el Departamento de Anatomía y Anatomía Comparadas de la Facultad de Veterinaria de Córdoba.

\section{Realización del diagnóstico histológico de los casos}

La actual clasificación histológica internacional de tumores de mama de los animales domésticos es la publicada por la Organización Mundial de la Salud en $1999^{25}$ y es la que se ha seguido en este estudio.

\section{Valoración de las variables invasión (estadio), grado de malignidad y tipo histológico}

Para la valoración de la variable invasión (estadio) se han adoptado los criterios de los autores Gilbertson y cols., 1983"5; para analizar el grado de malignidad los de Lagadic M, Estrada M, 199046, en la especie canina, y los de Castagnaro y cols., $1998^{47}$, en la felina; y para determinar el tipo histológico los de Misdorp y cols., $1999^{25}$.

\section{Valoración del porcentaje de casos positivos con cada uno de los marcadores tumorales utilizados}

Los RE y RP tienen un patrón de expresión nuclear y se consideran positivos lo casos con un porcentaje de células positivas mayor o igual al 5\% (Millanta y cols., 2005) ${ }^{38}$. El HER2 tiene un 
patrón de expresión de membrana citoplasmática y el umbral de positividad se sitúa en el 10\% de las células (Millanta y cols., $2005)^{38}$. Finalmente la CK 14 tiene un patrón de expresión citoplasmático y se consideran positivos lo casos con un porcentaje de células positivas mayor o igual al 1\% (Kim y cols., 2006 $)^{21}$.

\section{Relación entre los subtipos moleculares obtenidos mediante métodos inmunohistoquímicos y las variables invasión (estadio), grado de malignidad y tipo histológico}

Para ver si existen diferencias significativas en la distribución de los subtipos moleculares en cada uno de los grupos de las variables invasión, grado de malignidad y tipo histológico se ha utilizado el test de $\mathrm{X}^{2}$ de Pearson mediante programa estadístico STATA v.11.0. Intervalo de confianza del 95\%.

\section{RESULTADOS}

La clasificación histológica de los tumores analizados fue la siguiente: 6 carcinomas simples de tipo sólido (4 de perra y 2 de gata), 3 carcinomas simples de tipo tubulopapilar (perra), 1 tumor mixto maligno (perra), 1 carcinoma en adenoma (perra), 1 fibroadenoma atípico (gata) y un carcinoma simple tipo anaplásico (perra). A continuación aparecen microfotografías en HE de algunos de los tumores malignos diagnosticados. Ver figura 2.

La valoración del estadio de los tumores reveló la existencia de 1 carcinoma no infiltrante (gata), 9 tumores que invadían el estroma ( 7 de perra y 2 de gata) y 3 que además de invadir el estroma presentaban invasión vascular en forma de émbolos de células tumorales en los vasos (perra). Respecto a la valoración del grado de malignidad, 2 tumores fueron de grado I ( 1 de perra y 1 de gata), 6 de grado II (4 de perra y 2 de gata) y 4 de grado III (perra). En uno de ellos no procedía establecer el grado puesto que era un carcinoma anaplásico (perra). En la valoración del tipo histológico 10 tumores fueron de tipo simple ( 8 de perra y 2 de gata), 1 de tipo complejo o mixto (perra) y 2 fueron de otros tipos distintos a los dos anteriores (1 de perra y 1 de gata).

En la figura 3 se muestra un ejemplo de la valoración de la variable invasión (estadio). Se observa la presencia de émbolos de células tumorales en los vasos, indicativo de que este tumor está en el estadio 2 o de invasión vascular. La figura 4 es un ejemplo de valoración de la variable grado de malignidad. Se observa cómo las células tumorales forma túmulos ocasionalmente, el grado de anisocariosis es intensa y el número de mitosis en este campo de 400 aumentos es, por lo menos, de 4.

Según los criterios de clasificación respecto a la presencia o no de receptores hormonales, es decir, de RE y RP, 3/13 de los tumores expresaron RE y RP ( 2 de perra y 1 de gata) mientras que 10/13 fueron negativos ( 8 de perra y 2 de gata).

En cuanto a los resultados respecto a los criterios para establecer los subtipos moleculares, el 23\% (3/13) de los tumores fueron del subtipo luminal B ( 2 de perra y 1 de gata), el $23 \%$ (3/13) fueron HER2 positivos (2 de perra y 1 de gata), el $46 \%$ (6/13) fueron del subtipo basal (5 de perra y 1 de gata) y el 7,6\% (1/13) no se pudieron clasificar porque no expresaron ninguno de los marcadores tumorales analizados (perra). Ningún caso fue del subtipo luminal A.

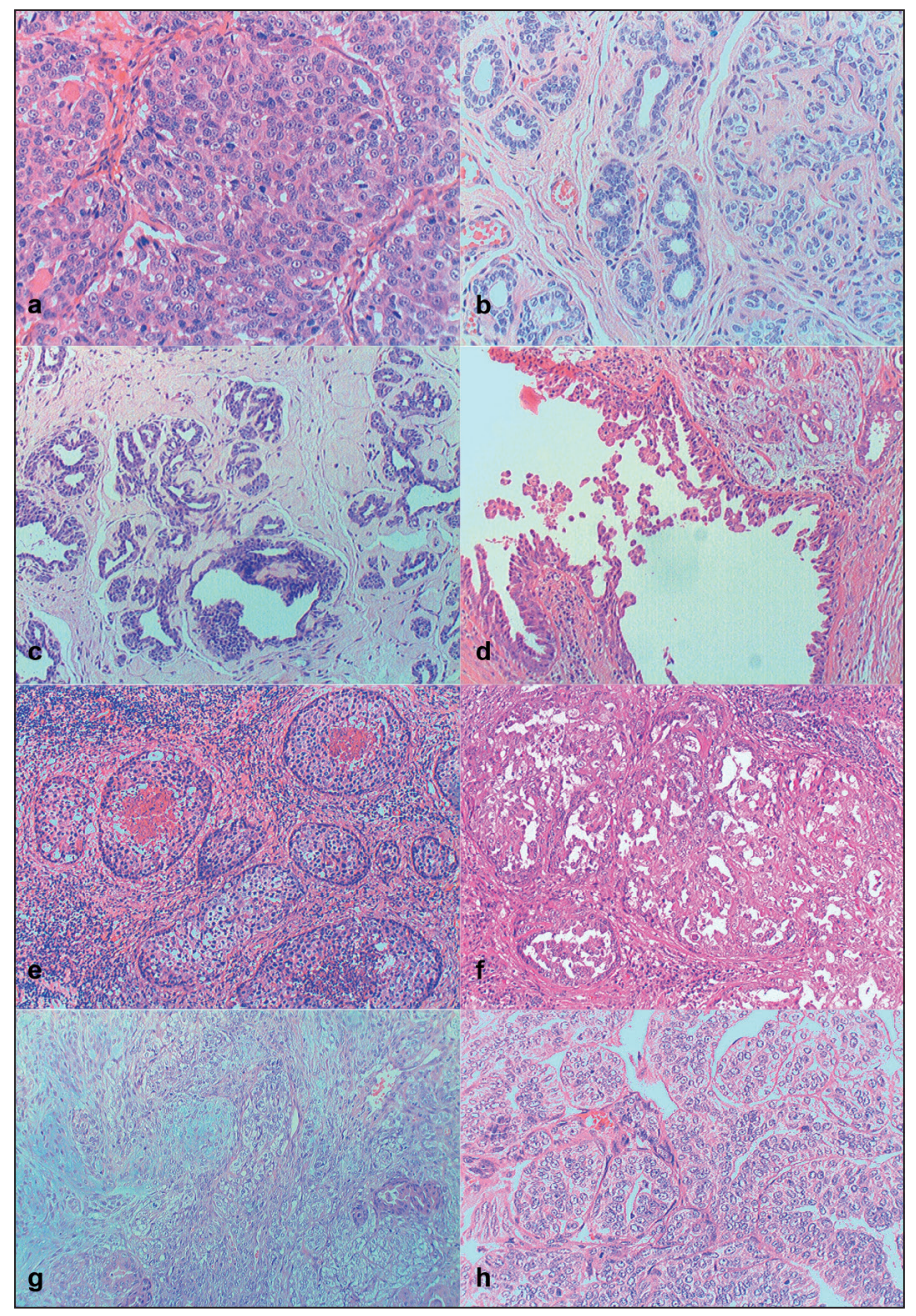

Figura 2. (a) Gata. Carcinoma sólido. HE x200. (b) Perra. Carcinoma en adenoma. HE x100. (c) Gata. Fibroadenoma atípico. HE x100. (d) Perra. Carcinoma anaplásico. HE x100. (e) Perra. Carcinoma sólido. HE x200. (f) Perra. Carcinoma tubulopapilar. HE x200. (g) Perra. Tumor Mixto Maligno. HE x100. (h) Perra. Carcinoma tubulopapilar. HE 2200 .

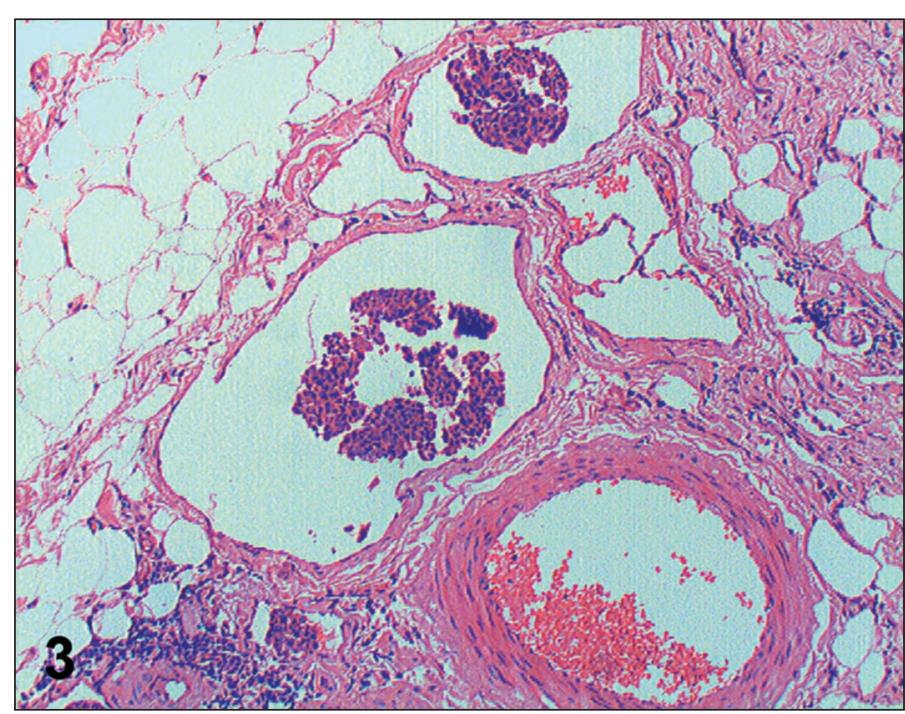

Figura 3. Invasión vascular. Perra. Carcinoma sólido. HE x200. 


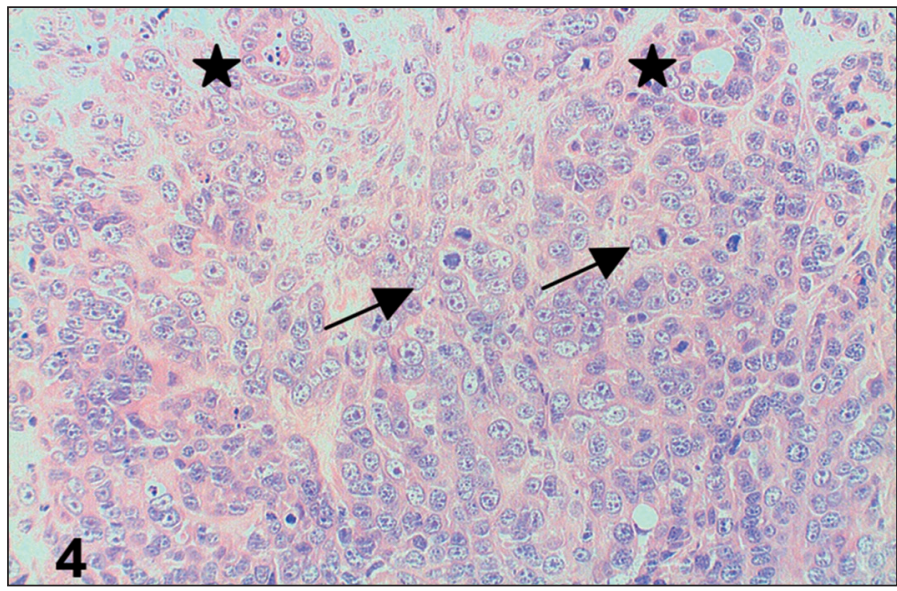

Figura 4. Perra. Carcinoma tubulopapilar. Grado III. Diferenciación tubular (asteriscos) y mitosis (flechas) HE x200.

Los controles internos fueron positivos para todos los marcadores moleculares.

De los 6 tumores clasificados como basales todos fueron de grado histológico II o III. Por otro lado, todos los tumores basales presentaban o infiltración de solo el estroma o también invasión vascular. Dos tercios de los tumores clasificados como HER2 positivos presentaban infiltración del estroma y uno de los 2 tumores en los que era posible valorar el grado de malignidad resultó ser de grado II. En cuanto al subtipo luminal B 2/3 eran de grado II o III y $1 / 3$ de grado I. Ver tabla 1 .

No se encontraron diferencias significativas en la distribución de los subtipos moleculares entre los diferentes grupos de la variable invasión ( $\mathrm{p}$-valor=0,26). Tampoco se observaron diferencias significativas en la distribución de los subtipos moleculares entre los diferentes grupos de la variable grado de malignidad ( $\mathrm{p}$-valor=0,42). Por el contrario, sí hubo diferencias en el límite de la significación estadística en la distribución de los subtipos moleculares entre los diferentes grupos de la variable tipo histológico $(\mathrm{p}=0,08)$. Ver tabla 2 .

\section{DISCUSIÓN}

En este estudio la mayoría de los tumores, 8 de perra y 2 de gata, (10/13) resultaron ser negativos a los receptores hormona-

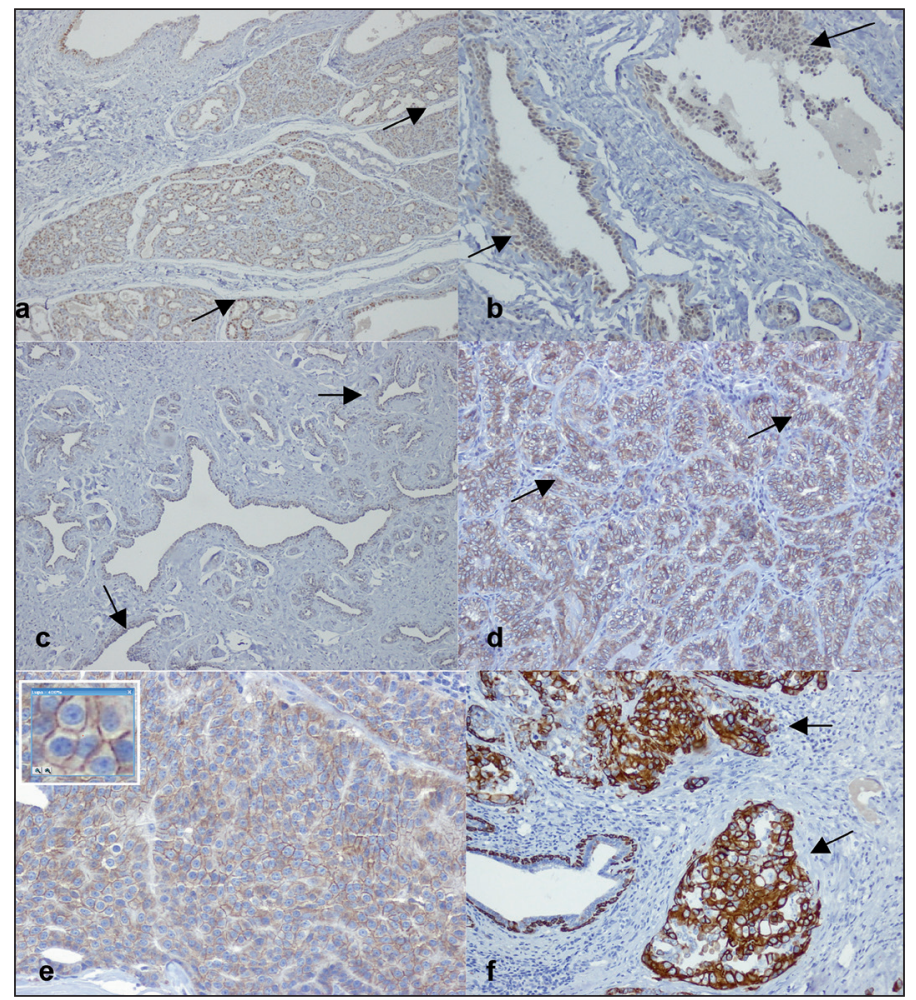

Figura 5. (a) Expresión de RE. Control interno (flechas). ABC $x 100$. (b) Expresión positiva al receptor de estrógenos. Zona tumoral (flechas). ABC x200. (c) Expresión positiva al receptor de progesterona. Zona tumoral (flechas). ABC x100. (d) Expresión positiva a HER2. Zona tumoral. ABC x200 (Inserción X400). (e) Expresión positiva a HER2. Zona tumoral (flechas). ABC x400. (f) Expresión positiva a CK14. Zona tumoral (flechas). $A B C \times 200$.

les, estrógenos y progesterona, mientras que en un estudio de Sassi y cols., $2010^{30}$, que realizó de forma similar con 45 casos de carcinomas de perra los negativos a hormonas fueron una minoría (10/45). Illera y cols., $2006^{48}$, en un estudio realizado con 32 tumores mamarios malignos no inflamatorios en perra, la mayoría fueron negativos al receptor de estrógenos (30/32, 93,70\%).

La obtención de cuatro subtipos moleculares de los cinco posibles en la perra y 3 en la gata, sugiere que los tumores ma-

Tabla 1. Resumen clasificación histológica, especie, positividad a receptores hormonales, subtipo molecular, invasión (estadio), grado y tipo histológico

\begin{tabular}{|cccccccc|}
\hline Muestra & Clasificación Histológica & Especie & RP/RE & Subt. molecular & Estadio & Grado & Tipo histológico \\
\hline 7205 & Carcinoma simple: sólido & Canina & Negativo & Basal & Vascular & II & Simple \\
11209 & Carcinoma simple: tubulopapilar & Canina & Negativo & Basal & Estroma & III & Simple \\
12027 & Carcinoma simple: sólido & Felina & Negativo & Basal & Estroma & II & Simple \\
11081 & Carcinoma simple: tubulopapilar & Canina & Negativo & Basal & Vascular & II & Simple \\
8099 & Carcinoma simple: sólido & Canina & Negativo & Basal & Estroma & III & Simple \\
8166 & Carcinoma simple: sólido & Canina & Negativo & Basal & Vascular & II & Simple \\
12029 & Carcinoma en adenoma & Canina & Positivo & Luminal B & Estroma & II & Otros \\
7229 & Fibroadenoma atípico & Felina & Positivo & Luminal B & Estroma & I & Otros \\
12021 & Carcinoma simple: tubulopapilar & Canina & Positivo & Luminal B & Estroma & III & Simple \\
6142 & Carcinoma simple: sólido & Felina & Negativo & HER2 & NI & II & Simple \\
8338 & Tumor mixto maligno & Canina & Negativo & HER2 & Estroma & I & Complejo/mixto \\
7446 & Carcinoma simple: anaplásico & Canina & Negativo & HER2 & Estroma & NP & Simple \\
7201 & Carcinoma simple: sólido & Canina & Negativo & Sin clasificar & Estroma & III & Simple \\
\hline
\end{tabular}

NI: No Infiltrante. NP: No Procede. 
Tabla 2. Subtipos moleculares frente a variables Invasión (estadio), grado de malignidad y tipo histológico

\begin{tabular}{|c|c|c|c|c|c|c|c|}
\hline Variables & Grupos & Luminal-tipo B & HER-2 & Basal & Sin clasificar & Total & p-valor \\
\hline \multirow{6}{*}{ Invasión (estadio) } & No infiltrante $(0)$ & 0 & 1 & 0 & 0 & 1 & ns \\
\hline & $\%$ & 0 & 100 & 0 & 0 & 100 & \\
\hline & Invasión del estroma (I) & 3 & 2 & 3 & 1 & 9 & ns \\
\hline & $\%$ & 33,33 & 22,22 & 33,33 & 11,11 & 100,00 & \\
\hline & Invasión vascular (II) & 0 & 0 & 3 & 0 & 3 & ns \\
\hline & $\%$ & 0 & 0 & 100 & 0 & 100 & \\
\hline \multirow[t]{6}{*}{ Grado } & I & 1 & 1 & 0 & 0 & 2 & $\mathrm{~ns}$ \\
\hline & $\%$ & 50,00 & 50,00 & 0,00 & 0,00 & 100,00 & \\
\hline & II & 1 & 1 & 4 & 0 & 6 & ns \\
\hline & $\%$ & 16,67 & 16,67 & 66,67 & 0,00 & 100,00 & \\
\hline & III & 1 & 0 & 2 & 1 & 4 & ns \\
\hline & $\%$ & 25,00 & 0,00 & 50,00 & 25,00 & 100,00 & \\
\hline \multirow[t]{6}{*}{ Tipo histológico } & Simple & 1 & 2 & 6 & 1 & 10 & $\mathrm{~ns}$ \\
\hline & $\%$ & 10,00 & 20,00 & 60,00 & 10,00 & 100,00 & \\
\hline & Complejo/mixto & 0 & 1 & 0 & 0 & 1 & $\mathrm{~ns}$ \\
\hline & $\%$ & 0,00 & 100,00 & 0,00 & 0,00 & 100,00 & \\
\hline & Otros & 2 & 0 & 0 & 0 & 2 & $\mathrm{~ns}$ \\
\hline & $\%$ & 100,00 & 0,00 & 0,00 & 0,00 & 100,00 & \\
\hline
\end{tabular}

ns: no significativo.

marios caninos y felinos son buenos modelos para el cáncer de mama en la especie humana, pero sería necesario repetir el estudio con un mayor número de casos y comparar entre grupos homogéneos. Sassi y cols., $2010^{30}$ obtuvieron 3 de 5 (luminal A, luminal B y basal) en la especie canina. Solo uno de los tumores fue del subtipo molecular sin clasificar ya que obtuvo un resultado negativo en todos los marcadores tumorales utilizados. Este resultado podría haberse relacionado con algún error durante el procesamiento de las muestras de tejido (el tiempo de fijación y la temperatura de la parafina influyen en la conservación de los antígenos y pueden llegar a destruirlos), sin embargo los controles internos fueron positivos para todos los receptores.

De los 6 tumores clasificados como basales todos fueron de grado II o III. Por otro lado, todos los tumores basales presentaban infiltración o de solo el estroma o también invasión vascular. Este resultado parece que se correlaciona con la pérdida de receptores hormonales que tiene lugar con la progresión del tumor, como señalan los estudios de Nieto y cols., $2000^{49}$ y Millanta y cols., $2005^{38}$.

Dos tercios de los tumores clasificados como HER2 positivos presentaban infiltración del estroma y 1 de los 2 en los que fue posible valorar el grado de malignidad era de grado II. Este resultado también parece relacionado con la sobreexpresión de HER2 observada en los tumores de mayor malignidad, como demuestran los trabajos de Martín de las Mulas y cols., $2003^{37}$ y Ahern y cols., $1996^{42}$.

No se encontraron diferencias significativas en la distribución de los subtipos moleculares entre los diferentes grupos de la variable invasión (estadio) al igual que en el estudio de Sassi y cols., $2010^{30}$. Tampoco se encontraron diferencias significativas en la distribución de los subtipos moleculares entre los diferentes grupos de la variable grado de malignidad. Por el contrario, Sassi y cols., $2010^{30}$, encontraron un alto porcentaje del subtipo luminal A y basal entre los carcinomas de grado I (luminal A 50\%, luminal B $12 \%$, basal 38\%); además, el porcentaje de los casos con subtipo luminal B fue mayor en el grado II (luminal A 14\%, luminal B $63 \%$, basal $23 \%$ ) y en el grado III (luminal A $15 \%$, luminal B $77 \%$, basal $8 \%$ ). En este estudio se encontraron diferencias en el límite de la significación estadística en la distribución de los subtipos moleculares entre los diferentes grupos de la variable tipo histológico. Sin embargo, Sassi y cols., $2010^{30}$ no encontraron diferencias significativas. A la hora de valorar los resultados del análisis estadístico, es necesario tener en cuenta el pequeño número muestras de las que se disponía a la hora de realizar nuestro estudio. Sin embargo, sí obtuvimos 2/3 luminal B entre los grados II y III.

Se trata de un estudio que se ampliará en un futuro con un mayor número de muestras y la inclusión de otras variables, como el tiempo libre de enfermedad, lo que permitirá evaluar además su relación con el pronóstico.

\section{CONCLUSIONES}

1. La aplicación del panel de anticuerpos normalmente utilizado para caracterizar los subtipos moleculares en los tumores de mama de la especie humana ha permitido identificar 4 (luminal B, HER2, basal y sin clasificar) en la perra y 3 (luminal B, HER2 y basal) en la gata de los 5 tipos posibles (ninguno ha sido luminal A). Esta clasificación de los tumores de mama en subtipos moleculares se podría usar en las especies canina y felina.

2. No se han encontrado diferencias significativas en la distribución de los subtipos moleculares en cada uno de los grupos de las variables invasión, grado de malignidad y tipo histológico. No existe asociación entre los subtipos moleculares y las variables invasión, grado de malignidad y tipo histológico.

\section{AGRADECIMIENTOS}

Estudio parcialmente financiado por el proyecto AGL201102553 del Ministerio de Innovación, Ciencia y Empresa, Gobierno de España.

\section{BIBLIOGRAFÍA}

1. Vos JH, van den Ingh TS, Misdorp W, Molenbeek RF, van Mil FN, Rutteman GR, Ivanyi D, Ramaekers FC. Immunohistochemistry with keratin, vimentin, desmin, and alpha-smooth muscle actin monoclonal antibodies in canine mammary gland: benign mammary tumours and duct ectasias. Vet $Q$ 1993; 15: 89-95. 
2. Griffey SM, Madewell BR, Dairkee SH, Hunt JE, Naydan DK, Higgins RJ.Immunohistochemical reactivity of basal and luminal epithelium-specific cytokeratin antibodies within normal and neoplastic canine mammary glands. Vet Pathol 1993; 30: 155-161.

3. Gärtner F, Geraldes M, Cassali G, Rema A, Schmitt F. DNA measurement and immunohistochemical characterization of epithelial and mesenchymal cells in canine mixed mammary tumours: putative evidence for a common histogenesis. Vet J 1999; 158: 39-47.

4. Reis-Filho JS, Tutt AN. Triple negative tumours: a critical review. Histopathology 2008; 52: 108-118.

5. Hellmén E, Lindgren A. The expression of intermediate filaments in canine mammary glands and their tumors. Vet Pathol 1989; 26: 420-428.

6. Destexhe E, Lespagnard L, Degeyter M, Heymann R, Coignoul F. Immunohistochemical identification of myoepithelial, epithelial, and connective tissue cells in canine mammary tumors. Vet Pathol 1993; 30: 146-154.

7. Gama A, Alves A, Gartner F, Schmitt F: p63: a novel myoepithelial cell marker in canine mammary tissues. Vet Pathol 2003; 40: 412-420.

8. Abd El-Rehim DM, Pinder SE, Paish CE, Bell J, Blamey RW, Robertson JF, Nicholson RI, Ellis IO. Expression of luminal and basal cytokeratins in human breast carcinoma. J Pathol 2004; 203: 661-671.

9. Sánchez-Céspedes R, Millán Y, Guil-Luna S, García-Monterde J, Reymundo C, Espinosa de los Monteros A, Martín de las Mulas J. Myoepithelial cell layer integrity in canine mamary carcinoma. J Comp Path 2011; 145: 25-30.

10. Chu P, Wu E, Weiss, LM. Cytokeratin 7 and cytokeratin 20 expression in epithelial neoplasms: a survey of 435 cases. Mod Pathol 2000; 13: 962-972.

11. Barak V, Goike H, Panaretakis KW, Einarsson R. Clinical utility of cytokeratins as tumor markers. Clin Biochem 2004; 37: 529-540.

12. Bonnett BN, Egenvall A, Hedhammar A, Olson P. Mortality in over 350,000 insured Swedish dogs from 1995-2000: I. Breed-, gender-, age- and causespecific rates. Acta Vet Scand 2005; 46 (3): 105-20.

13. Ettinger SJ. Textbook of Veterinary Internal Medicine. 2 ed. Philadelphia: W.B. Saunders Company, 1995: 412-413.

14. Baba AI, Câtoi C. Comparative Oncology. Bucharest: The Publishing House of the Romanian Academy, 2007: 87-407.

15. Millanta F, Lazzeri G, Mazzei M, Vannozzi I, Poli A. MIB-1 Labeling indexin feline dysplastic and neoplastic mammary lesions and its relationship with post surgical prognosis. Veterinary Pathology 2002; 39: 120-126.

16. Perou CM, Sorlie T, Eisen MB, Rijn van de M, Jeffrey SS, Rees CA, Pollack JR, Ross DT, Johnsen H, Akslen LA, Fluge O, Pergamenschikov A, Williams C, Zhu SX, Lønning PE, Børresen-Dale AL, Brown PO, Botstein D. Molecular portraits of human breast tumours. Nature 2000; 406: 747-752.

17. Sørlie T, Tibshirani R, Parker J, Hastie T, Marron JS, Nobel A, Deng S, Johnsen H, Pesich R, Geisler S, Demeter J, Perou CM, Lønning PE, Brown O, BørresenDale A-L, Botstein D. Repeated observation of breast tumor subtypes in independent gene expression data sets. Proc Natl Acad Sci USA 2003; 100: 8418-8423.

18. Sørlie T, Wang Y, Xiao C, Johnsen H, Naume B, Samaha RR, Børresen-Dale A-L: Distinct molecular mechanisms underlying clinically relevant subtypes of breast cancer: gene expression analyses across three different platforms. BMC Genomics 2006; 7:127.

19. Fan C, Oh DS, Wessels L, Weigelt B, Nuyten DS, Nobel AB, van't Veer LJ, Perou $\mathrm{CM}$. Concordance among gene-expression-based predictors for breast cancer. N Engl J Med 2006; 355: 560-569.

20. Conforti R, Boulet T, Tomasic G, Taranchon E, Arriagada R, Spielmann M, Ducourtieux M, Soria JC, Tursz T, Delaloge S, Michiels S, Andre F. Breast cancer molecular subclassification and estrogen receptor expression to predict efficacy of adjuvant anthracyclines-based chemotheraphy: a biomarker study from two randomized trials. Ann Oncol 2007; 18(9): 1477-1483.

21. Kim MJ, Ro JY, Ahn SH, Kim HH, Kim SB, Gong G. Clinicopathologic significance of the basal-like subtype of breast cancer: a comparison with hormone receptor and Her2/neu-overexpressing phenotypes. Hum Pathol 2006; 37: 1217-1226.

22. Cheang MC, Voduc D, Bajdik C, Leung S, McKinney S, Chia SK, Perou CM, Nielsen TO: Basal-like breast cancer defined by five biomarkers has superior prognostic value than triple-negative phenotype. Clin Cancer Res 2008; 14(5): 1368-1376.

23. Misdorp W: Quelques aspects comparatifs des cancers de la mammelle chez la chienne, la chatte et la femme. Rec Med Vet Ec Alfort 1972; 148: 583-590.

24. Misdorp W, Weijer K . Animal model of human disease: breast cancer. Am J Pathol 1980; 98: 573-576.

25. Misdorp W, Esle RW, Hellmén E, Lipscomb TP: Histological Classification of Mammary Tumors of the Dog and Cat. Published by the Armed Forces Institute of Pathology in cooperation with the American Registry of and the
World Health Organization Collaborating Centre for Worldwide Aderence on Comparative Oncology. Washington, DC 1999.

26. Rosen PP. Myoepithelial Neoplasms. Rosen's breast pathology Lippincott Williams \& Wilkins, Philadelphia, PA, 2, 2001: 425-453.

27. Brodey RS, Goldschmidt MH, Roszel JR. Canine mammary gland neoplasms. J Am Anim Hosp Assoc 1983; 19: 61-90.

28. Gama A, Alves A, Schmitt F. Identification of molecular phenotypes in canine mammary carcinomas with clinical implications: application of the human classification. Virchows Arch 2008; 453:123:32.

29. Gama A, Alves A, Schmitt F. Expression and prognostic significance of CK19 in canine malignant mammary tumours. Vet J 2010; 184: 45-51.

30. Sassi F, Benazzi C, Castellani G, Sarli G. Molecular-based tumour subtypes of canine mammary carcinomas assessed by immunohistochemistry. BMC Vet Res 2010; 6: 5 .

31. Chang CC, Tsai MH, Liao JW, . Evaluation of hormone recepto expression for use in predicting survival of female dogs with malignant mammary gland tumours. J Am Vet Med Assoc 2009; 235 (4): 391-396.

32. Martín de las Mulas J, Millán Y, Rios R. A prospective análisis of immunohistochemically determined estrogen receptor alfa and progesterona receptor expresión and host and tumour factors as predictors of disease free period in mamary tumors of the dog. Vet pathol 2005, 42: 200-2012.

33. Slamon DJ, Clark GM, Wong SG, Levin WJ, Ullrich A, McGuire WL. Human breast cancer: correlation of relapse and survival with amplification of the HER-2/neu oncogene. Sciencie 1987; 235: 177-12.

34. Sahin AA. Biologic and clinical significance of HER-2/neu (cerbB-2) in breast cancer. Adv Anat Pathol 2000; 7: 158-166.

35. Rungsipipat A, Tateyama S, Yamaguchi R, Uchida K, Miyoshi N, Hayashi T. Immunohistochemical analysis of c-yes and c-erbB-2 oncogene products and 53 tumor suppressor protein in canine mammary tumors. J Vet Med Sci 1999; 61: 27-32.

36. Dutra AP, Granja NV, Schmitt FC, Cassali GD. c-erbB-2 expression and nuclear pleomorphism in canine mammary tumors. Braz J Med Biol Res 2005; 37: $1673-1681$

37. Martín de las Mulas J, Ordás J, Millán Y, Fernández-Soria V, Cajal SR. Oncogene HER-2 in canine mammary gland carcinomas. Breast Cancer Res Treat 2003; 80: 363-367.

38. Millanta F, Calandrella M, Bari G, Niccolini M, Vannozzi I, Poli A. Comparison of steroid receptor expression in normal, dysplastic, and neoplastic canine and feline mammary tissues. Res Vet Sci 2005; 79: 225-232.

39. Winston J, Craft DM, Scase TJ, Bergman PJ. Immnunohistochemical detection of HER-2 expression in spontaneous feline mammary tumors. VCO 2005; 3: 8-15.

40. De María R, Olivero M, Lussich S, Nakaichi M, Murata T, Biolatti B, Di Renzo F. Spontaneous feline mammary carcinoma is a model of HER2 overexpressing poor prognosis human breast cancer. Cancer Res 2005; 65: 907-912.

41. Ordás J, Millán Y, Dios R, Reymundo C, Martín de las Mulas J. Proto-oncogen HER-2 in normal, dysplastic and tumorous felina mammary glands: an immunohistochemical and chromogenic in situ hybridization study. BMC Cancer 2007; 7: 179

42. Ahern TE, Bird RC, Bird AE, Wolfe LG. Expression of the oncogene cERBB-2 in canine mammary cancers and tumor-derived cell lines. Am J Vet Res 1996; 57(5): 693-696.

43. Rutteman GR, Withrow SJ, MacEwen EG. Tumors of the mammary gland. In Small Animal Clinical Oncology. En: Withrow SJ, MacEwen BR (eds). Philadelphia: W.B. Saunders Company, 2001: 455-477.

44. Galán Torres JA. Seminario sobre Técnica Inmunohistoquímica. Centro Militar de Veterinaria de la Defensa. Madrid, España: 2002.

45. Gilbertson SR, Kurzman ID, Zachrau RE, Hurvitz AI, Black MM. Canine mammary epithelial neoplasms: biological implications of morphologic characteristics assessed in 232 dogs. Vet Pathol 1983; 20: 127-142.

46. Lagadic M, Estrada M. Tumeurs mammaires de la chienne: critères du prognostic histologique et interèt d'un grading. Rec Med Vet Ec Alfort 1990; 166(11): 1035-1042.

47. Castagnaro M, Casalone C, Bozzetta E, De Maria R, Biolatti B, Caramelli M. Tumour Grading and the one-year post surgical prognosis in feline mammary carcinoma. J Comput Pathol 1998; 119: 263-275.

48. Illera JC, Pérez-Alenza MD, Nieto A, Jiménez MA, Silvan G, Dunner S, Peña L. Steroids and receptos in canine mammary cancer. Steroids 2006; 71: 541-548.

49. Nieto A, Peña L, Pérez-Alenza MD, Sànchez MA, Flores JM, Castanõ M Immunohistologic detection of estrogen receptor alpha in canine mammary tumors: clinical and pathologic associations and prognostic significance. Vet Pathol 2000, 37: 239-247. 